\title{
Scaling Properties of Diffusion-Limited Reactions on Fractal and Euclidean Geometries
}

\author{
Katja Lindenberg, ${ }^{1}$ Wen-Shyan Sheu, ${ }^{1,2}$ and Raoul Kopelman ${ }^{3}$
}

\begin{abstract}
We review our scaling results for the diffusion-limited reactions $A+A \rightarrow 0$ and $\mathrm{A}+\mathrm{B} \rightarrow 0$ on Euclidean and fractal geometries. These scaling results embody the anomalies that are observed in these reactions in low dimensions; we collect these observations under a single phenomenological umbrella. Although we are not able to fix all the exponents in our scaling expressions from first principles, we establish bounds that bracket the observed numerical results.
\end{abstract}

KEY WORDS: Diffusion-limited reactions; fractals; scaling; aggregates.

\section{INTRODUCTION}

The kinetic laws that describe diffusion-controlled annihilation reactions of the form $\mathrm{A}+\mathrm{A} \rightarrow 0$ and $\mathrm{A}+\mathrm{B} \rightarrow 0$ in low dimensions differ from the "classical" mean field forms and are frequently called "anomalous." The so-called anomalous behavior arises from the fact that diffusion in low dimensions is not an effective mixing mechanism; consequently, the spatial distributions of reactants differ from the thoroughly mixed ones that are implicit in the classical rate laws.

It is useful to begin by describing what we mean by "thoroughly mixed." In the single-species case $\mathrm{A}+\mathrm{A} \rightarrow 0$, thorough mixing implies a distribution of nearest-neighbor distances of the Hertz form. ${ }^{(1)}$ This distribution includes small nearest-neighbor distances with a high probability; as nearby reactants annihilate one another, an effective mixing mechanism

\footnotetext{
${ }^{1}$ Department of Chemistry, 0340, and Institute for Nonlinear Science, 0402, University of California at San Diego, La Jolla, California 92093.

${ }^{2}$ Current address: Department of Chemistry, University of Texas, Austin, Texas 78712.

${ }^{3}$ Departments of Chemistry and Physics, University of Michigan, Ann Arbor, Michigan 48109.
} 
replenishes them very quickly. For the $A+B \rightarrow 0$ reaction, thorough mixing implies not only a Hertz distribution of nearest neighbor distances at all times, but also a spatial distribution of A's and B's that is totally random. In the $\mathrm{A}+\mathrm{A} \rightarrow 0$ reaction, anomalous behavior is observed when the distribution of A's deviates from the Hertz form. ${ }^{(1)}$ In the $\mathrm{A}+\mathrm{B} \rightarrow 0$ reaction, anomalies arise when, in addition to deviations from the Hertz distribution, nearby unequal pairs are depleted and not replenished, leading to the formation of aggregates of like particles ${ }^{(2-4)}$ (for a review, see, e.g., ref. 2).

The precise way in which the anomalous kinetic laws deviate from the classical behavior, and the critical dimension above which the behavior is classical, depend sensitively on the detailed conditions at hand. ${ }^{(111)}$ These include the nature of the reaction (e.g., $\mathrm{A}+\mathrm{A} \rightarrow 0$ or $\mathrm{A}+\mathrm{B} \rightarrow 0$ ), the initial spatial distribution of reactants, the absence or presence of reactant sources and their spatial and temporal statistics, and whether the system is finite or infinite. The approaches that have been used to arrive at these results include extensive numerical simulations ${ }^{(12-16)}$ and a number of theoretical methods. ${ }^{(2-11,17)}$ The latter include a variety of truncated particle-distribution hierarchies, ${ }^{(2,7,8,17,18)}$ a small number of exact results, ${ }^{(10,19)}$ reactiondiffusion equation models (which can be viewed as special cases of the hierarchy approaches), ${ }^{(6)}$ and scaling arguments. ${ }^{(9,11,20)}$ Although reactiondiffusion models have been extremely useful for the analysis of these reactions on Euclidean geometries, the fact that a simple second-order diffusion equation is not appropriate to describe transport on fractal structures ${ }^{(21)}$ has limited the applicability of these models. In fact, few theoretical attempts (even at the phenomenological level) have been made to deal with even these simple reactions on fractals. ${ }^{(8,9,20,22)}$

Scaling arguments have proven extremely useful toward understanding the kinetic behavior in the anomalous regime. ${ }^{(5,9,11,20)}$ Although scaling arguments do not replace first-principles approaches and only yield unequivocal results in situations where various exponents are known from other theories, they do offer a simple way of categorizing and parametrizing the problem. This allows one to express the "unknown" features in terms of a few parameters (typically, exponents) that must either be fixed through other theories or otherwise conjectured.

We have developed scaling descriptions for the diffusion-limited reactions $\mathrm{A}+\mathrm{A} \rightarrow 0$ and $\mathrm{A}+\mathrm{B} \rightarrow 0$ on Euclidean and fractal lattices. ${ }^{(9,20)}$ An important consequence of our approach is an indication that the anomalous kinetic laws may at least in some cases depend not only on the spectral dimension, but also on other system dimensions, including the fractal dimension. In this paper we present our scaling formulas and suggest possible extensions of the applicability of our arguments to situa- 
tions that had not previously been included under this single umbrella. Since at least some of the exponents that we need as input in our equations have only been determined for systems of spectral dimension $d_{s}$ smaller than 2 , we restrict our remarks to this case.

The paper is organized as follows: In Section 2 we present a generalized form of our previous scaling relations that allows us to broaden their range of applicability. Section 3 then deals with the exponents that describe a variety of specific applications. For some of these cases, the kinetic laws have been obtained by other methods and we compare our results with them; in others, numerical simulations exist for comparison, while in yet other cases our results constitute a possible description of behavior that has not yet been checked by other procedures. In Section 4 , we comment on effects of correlated initial conditions and of the effects of sources. Section 5 is a brief summary of our conclusions. Some observations about the relation between our work and the recent results of Leyvraz and Redner ${ }^{(11,23)}$ are presented in the Appendix.

\section{SCALING RELATIONS}

We begin by restricting our analysis to irreversible reactions in the absence of sources except for the initial reactants, with equal initial concentrations of reactants. Our aim is to determine the exponent $\alpha$ in the decay law

$$
\rho \sim t^{-x}
$$

where $\rho$ is the global density of each reactant [our restriction to equal initial reactant concentrations leads to the form (2.1) rather than an exponential form]. For this purpose we introduce four quantities that we relate to one another and ultimately to the density and/or time in order to determine the desired exponent. ${ }^{(9,20)}$ The distinction between the reactions $\mathrm{A}+\mathrm{A} \rightarrow 0$ and $\mathrm{A}+\mathrm{B} \rightarrow 0$ will be made as needed.

The first quantity that we introduce is the reaction rate $R$; the second is $\tau$, the average time that it takes a particle to reach a nearest neighbor; the third is $N$, the number of nearest pairs in the system, regardless of particle identity. In these definitions we ignore differences between the average nearest-neighbor distances between particles of the same species and between particles of different species (see the Appendix for some comments on the effects of "depletion zones" that may introduce such differences $\left.^{(11,23)}\right)$. The fourth quantity, which we call $\chi$, is a measure of the fraction of the particles that are "isolated" and that can therefore not react with any nearest neighbor (because the nearest neighbors are all of the wrong type). 
Thus, $\chi^{-1}$ is proportional to the probability that a particle has an "unobstructed" nearest neighbor with which it can react, and $N / \chi$ is the number of nearest-neighbor pairs that can react. In the $\mathrm{A}+\mathrm{A} \rightarrow 0$ case there are no isolated particles, since all nearest neighbors can react with one another, and hence $\chi=1$, but in the $\mathrm{A}+\mathrm{B} \rightarrow 0$ reaction, deviations of $\chi$ from unity reflect the presence of aggregates of like particles. These four quantities are connected via the relation ${ }^{(9,20)}$

$$
R \sim N \times \frac{1}{\tau} \times \frac{1}{\chi}
$$

Thus, the rate of reaction is determined by the number of nearest-neighbor pairs, by the average rate at which a particle reaches a nearest neighbor, and is reduced if these nearest neighbors can in fact not all react with one another.

Next we relate each of these quantities to density and/or time. The time dependence of the reaction rate is clearly

$$
R=\dot{\rho} \sim t^{-\alpha-1}
$$

Since the number of nearest neighbors of each particle is of $O(1)^{\circ}$ (e.g., in a perfect lattice it would be of the order of the coordination number of the lattice), the number of nearest-neighbor pairs in the system is simply proportional to the number of particles, i.e.,

$$
N \sim \rho
$$

To find the density dependence of the time $\tau$, we argue as follows: We introduce $S_{l}$, the distinct number of sites visited by a walker in a time interval $t$. The distinct number of sites visited by a particle until it reaches its nearest neighbor in a compact random walk is of the order of the inverse of the particle density:

$$
S_{\tau} \sim \rho^{-1}
$$

On the other hand, the time that it takes a walker to visit this number of sites depends on the spectral dimension $d_{s}$ and can be found from the well-known relation between the distinct number of sites visited and the elapsed time $t$. For $d_{s} \leqslant 2$ (compact walk), the relation is ${ }^{(24)}$

$$
S_{t} \sim t^{d_{s} / 2}
$$

If we set the elapsed time equal to the average time $\tau$ that it takes a particle to reach a nearest neighbor and combine (2.6) with (2.5), we obtain

$$
\tau \sim \rho^{-2 / d_{s}}
$$


Finally, we consider the factor $\chi$. As stated above, this factor is unity in the $\mathrm{A}+\mathrm{A} \rightarrow 0$ case. For $\mathrm{A}+\mathrm{B} \rightarrow 0$, however, $\chi$ is in general different from unity when the species segregate into aggregates of like particles. To specify the form of $\chi$, we introduce a length $A$ as a measure of the average linear size of an aggregate of like particles. The number of like particles in an aggregate is then of order $A^{d_{f}}$, where $d_{f}$ is the fractal dimension of the system. The fraction $\chi$ of this number that is isolated (in the sense that all its nearest neighbors are particles of the same species) in general depends on the size of the aggregate. The detailed dependence on the size is determined by the nature of the system and by the configurations of the aggregates. We embody this dependence in the expression

$$
\chi \sim A^{\gamma}
$$

where $\gamma$ is an exponent that is as yet unspecified. In particular, the dependence of $\gamma$ on the various system dimensions (fractal, spectral, chemical, etc.) is in general unknown. Thus, this is the exponent that parametrizes our scaling expressions. To complete the relationships between density and time, we specify the way in which the linear size $A$ of an aggregate grows with time. For $d_{s}<2$ the exploration of a random walker is compact, i.e., the random walker covers essentially every site of a volume before leaving that volume; hence the linear size of an aggregate at time $t$ is the same as the distance a random walker has explored in time $t$. From Eq. (2.6) we then have

so that

$$
S \sim A^{d_{f}} \sim t^{d_{s} / 2}
$$

$$
\Lambda \sim t^{d_{s} / 2 d_{f}}
$$

We now combine Eqs. (2.3), (2.4), (2.7), (2.8), and (2.10) to obtain for the exponent $\alpha$ the expression ${ }^{(20)}$

$$
\alpha=\frac{d_{s}}{2}\left(1-\gamma \frac{d_{s}}{2 d_{f}}\right)
$$

This is our general result. In the next section we specialize the exponent $\alpha$ by choosing particular forms for $\gamma$ appropriate to different specific situations.

\section{SPECIFIC CASES}

Equation (2.11) is a general scaling result that must be specialized to particular situations through specific choices of the exponent $\gamma$. We begin 
by considering the simplest situation, namely, the case $\mathrm{A}+\mathrm{A} \rightarrow 0$. Here $\chi=1$ and hence

$$
\gamma=0
$$

so that

$$
\alpha=d_{s} / 2
$$

This exponent, which depends only on the spectral dimension, is well known from other treatments of the problem. ${ }^{(5,25)}$ The critical dimension for anomalous behavior is $d_{s}=2$; for spectral dimensions lower than this critical dimension, the decay of the concentration of reactant is slower than the classical prediction. This in turn reflects the deviation of the nearestneighbor distance distribution from the Hertz form..$^{(1,16)}$ The same behavior would obviously be observed in the A-B system if in addition to the A-B annihilation one would permit $\mathrm{A}-\mathrm{A}$ and $\mathrm{B}-\mathrm{B}$ annihilations to occur.

The next situation we consider is that of the $\mathrm{A}+\mathrm{B} \rightarrow 0$ reaction on Euclidean geometries with random initial conditions, i.e., the reactants are randomly deposited on the Euclidean system at time $t=0$. For these geometries the various dimensions are all equal, i.e., $d_{s}=d_{f}=d$. An initial random distribution implies that the initial density of each species is not uniform. Instead, there are local fluctuations such that one species is locally in excess of the other. As the reaction proceeds, the local "majority" species eliminates the "minority species"; as a consequence, aggregates of like particles are formed and grow in size with time. ${ }^{(2-4)}$ Reactions then only occur on the surfaces of these like-particle aggregates. If the interaggregate "reaction surfaces" are smooth ${ }^{(9,23)}$ then the ratio of inactive particles (i.e., those in the interior of the aggregates) to active ones (i.e., those on the surfaces of the aggregates) is roughly the volume-to-surface ratio

$$
\chi \sim A^{d} / A^{d-1}=\Lambda
$$

whence

$$
\gamma=1
$$

For the exponent $\alpha$ in (2.1) we then obtain the standard result also found using a number of other methods, ${ }^{(1-8,17)}$

$$
\alpha=d / 4
$$

The critical Euclidean dimension for anomalous behavior is thus $d=4$. We refer the reader to the Appendix for a discussion of an alternative analysis of this problem in Euclidean systems that has recently appeared in the 
literature, and which takes into account the formation of "depletion zones" between aggregates in which the densities are lower than in the interior of the aggregates. ${ }^{(11,16,23,26)}$

The two cases considered above reproduce well-known results. Consider now a situation which is less clear: that of the $\mathrm{A}+\mathrm{B} \rightarrow 0$ reaction on fractal geometries. A number of conjectures and simulation results for such systems are beginning to appear in the literature, ${ }^{(8,9,12,20,22)}$ but the behavior is not yet well understood. In particular, consider a random initial distribution of $\mathrm{A}$ and $\mathrm{B}$ particles. It has been conjectured (see, e.g., ref. 12 and references therein) that the result (3.5) can be extended to fractal geometries if one replaces the Euclidean dimension $d$ with the spectral dimension

$$
\alpha=d_{s} / 4
$$

According to this conjecture, the exponent $\alpha$ is independent of the fractal dimension. There are several reasons given for this conjecture, none of which appears firmly compelling. The analytic reasoning for this assumption tends to make use of diffusion arguments and scaling arguments that are certainly appropriate for Euclidean geometries, but whose applicability to fractal geometries is less certain. Numerical simulations produce results that might be consistent with this conjecture, but that may equally well arise in a different way (cf. below).

Instead of the direct extension (3.6), we envision several possible situations, depending on the nature of the fractal and of the aggregates. One situation might occur on random fractals in which the like-particle aggregates are again surrounded by smooth surfaces, a situation resembling that of the Euclidean geometries. If the particles on these surfaces can react with surface particles of neighboring aggregates, i.e., if they can reach one another in time $\tau$, then the ratio $\chi$ is again the volume-to-surface ratio (but now involving the fractal dimension),

$$
\chi \sim A^{d_{f}} / \Lambda^{d_{f}-1}=\Lambda
$$

so that again

$$
\gamma=1
$$

However, instead of Eq. (3.6) we now obtain

$$
\alpha=\frac{d_{s}}{2}\left(1-\frac{d_{s}}{2 d_{f}}\right)
$$

The exponent $\alpha$ thus depends on both the spectral and the fractal dimensions, and reduces to (3.5) or (3.6) only for Euclidean geometries. 
A second situation arises if the aggregates are not as smooth as they would be in a Euclidean geometry, but instead are fairly irregular and interpenetrating. The fraction of sites that is isolated may now be smaller than before, and might in general grow with $A$ according to a power which is smaller than unity:

$$
\gamma \leqslant 1
$$

The exponent $\alpha$ in this case would be larger than (3.9).

An extreme situation may occur for finitely-ramified fractals. In such fractals, aggregates of any size may actually have only a few points of contact with other aggregates, i.e., it may be possible to "cut" an aggregate (even a macroscopic one) out of the system by severing only a few connections. An example of a fractal in which the reaction surfaces that separate aggregates of different particles lie at such special nodes is a Sierpinski gasket in which a single aggregate of like particles occupies an entire triangular lobe. ${ }^{(13)}$ If this is the case, then essentially all the particles in the aggregate are unable to participate in the reaction, and the relative number of inactive to active particles is proportional to the volume of the aggregate:

so that

$$
\chi \sim \Lambda^{d_{f}} / \Lambda^{0}=\Lambda^{d_{f}}
$$

$$
\gamma=d_{f}
$$

Then

$$
\alpha=\frac{d_{s}}{2}\left(1-\frac{d_{s}}{2}\right)
$$

On the other hand, even on such fractals the reaction surfaces that separate aggregates may not be limited to these special nodes, and the reaction surfaces may then depend on the size of the aggregate. In this case, the ratio of inactive to active particles is smaller than (3.10) and the exponent $\gamma$ is smaller than $d_{f}$. A reasonable assumption might be that the reaction surfaces in this latter case behave as they do on a random fractal or on a Euclidean geometry, so that Eqs. (3.8) and (3.9) describe the situation. In fact, due to the hierarchical nature of some regular fractals such as Sierpinski gaskets one can imagine that the behavior of the reaction surfaces might oscillate between these two limiting behaviors (in effect causing the exponent $\alpha$ itself to be time-dependent) as the aggregates grow to occupy entire lobes of ever lower generations. The exponent $\alpha$ might then oscillate within the region defined by the limits

$$
\frac{d_{s}}{2}\left(1-\frac{d_{s}}{2}\right) \leqslant \alpha \leqslant \frac{d_{s}}{2}\left(1-\frac{d_{s}}{2 d_{f}}\right)
$$


Recent numerical simulations of Argyrakis and Kopelman on twoand three-dimensional critical percolation clusters ${ }^{(15,16)}$ yield a constant exponent $\alpha$ over several decades of time that lies within the bounds given by (3.14); the observed exponent in both cases is $\alpha=0.36$, while (3.14) gives $0.22 \leqslant \alpha \leqslant 0.43$ in two dimensions and $0.22 \leqslant \alpha \leqslant 0.49$ in three dimensions. With $d_{s}=4 / 3$, Eq. (3.6) gives $d_{s} / 4=0.33$. Whether these results confirm one or the other theory is still a matter of discussion.

Recent simulations of Zumofen et al. ${ }^{(12)}$ show for several multidimensional Sierpinski gaskets for times between $10^{2}$ and $10^{5}$ in units of steps that the exponent $\alpha$ oscillates within the bounds given by (3.14). A number of additional features of their oscillatory results are noteworthy and are consistent with our description. The reported oscillations drive the value of $\alpha$ below the "traditional value" (3.6), a behavior entirely consistent with our bounds. Our results also suggest a reason for the observed increase in the amplitudes of the oscillations with increasing spectral dimension: this amplitude may reflect the increasing difference between the upper and lower bounds in (3.14). Finally, our interpretation of the oscillations is consistent with the observation that the number of oscillations is smaller than the number of generations of the Sierpinski gasket, as expected within a finite simulation time. We note that the traditional exponent $d_{s} / 4$ is within the range included in (3.14) and that the "average" exponent may in fact be close to this value.

\section{CORRELATED INITIAL CONDITIONS AND SOURCES}

In this section we briefly reiterate for the sake of completeness some relevant results that we obtained earlier concerning the effects of correlated initial conditions, ${ }^{(27)}$ a situation for which no one has ventured a conjecture as to the behavior of the reaction rate in fractal systems. We also comment on the effects of external sources and the nature of the steady state that is achieved in this case. ${ }^{(27)}$

Consider an initial distribution of reactants that is correlated (rather than random) in the sense that initially $\mathrm{A}-\mathrm{B}$ pairs are constrained to lie within a finite average distance of each other. In previous work we dealt with this problem in Euclidean geometries on the basis of a reaction-diffusion model, ${ }^{(27)}$ and we found an exponent $\alpha$ that is actually larger than the corresponding exponent $\alpha=d / 2$ for the $\mathrm{A}+\mathrm{A} \rightarrow 0$ reaction [cf. Eq. (3.2)]:

$$
\alpha=(d+2) / 2
$$

This result seems puzzling at first glance; one might expect the $A+A \rightarrow 0$ exponent to be larger than the $A+B \rightarrow 0$ exponent regardless of initial 
condition, since in the former all nearest neighbors can react, while in the latter they cannot. However, this simple argument does not take the actual spatial distribution of reactants into account; our scaling formulas can be used to clarify the situation. The analysis in this case is made more transparent if we reexpress the behavior of the ratio $\chi$ of inactive to active particles in terms of time, using Eqs. (2.8) and (2.10). For Euclidean geometries we have

$$
\chi \sim t^{\gamma / 2}
$$

Imagine, for example, the following extreme situation in one dimension: A-B pairs of particles are placed at random exactly a distance $c$ lattice sites apart, with B always to the right of A. Initially the largest aggregates that are possible on the line are of size $c$. As time increases and the reaction proceeds, the particles at the ends of the aggregates react, and the number of particles per aggregate necessarily decreases. To reflect this shrinkage, the exponent in (4.2) must be negative, i.e., $\gamma<0$, since the ratio of inactive to active particles decreases. It is difficult $a$ priori to predict the value of the exponent from scaling arguments (although it should be possible in the example constructed above), but the result (4.1) is recovered if

$$
\gamma=(d-2) / d
$$

Notice that this result predicts a shrinkage in one dimension, but not in two, where the exponent $\alpha$ reduces to the "classical" value $\alpha=1$, as does that of the $\mathrm{A}+\mathrm{A} \rightarrow 0$ reaction. Thus, as time proceeds and the aggregates shrink, more reaction surfaces are created relative to the number of isolated particles and hence the rate of the reaction increases relative to that of the $A+A \rightarrow 0$ reaction (where there are no isolated particles) and to that of the $\mathrm{A}+\mathrm{B} \rightarrow 0$ reaction with random initial conditions (where the reaction surfaces relative to the number of isolated particles decrease as the aggregates grow in size).

It is interesting to speculate on the behavior of the corresponding exponents in fractal systems. This behavior has not been conjectured by any theory that we are aware of, and neither are we prepared to do so at this time.

In the presence of sources we have previously considered the balance between the continual input of reactants at rate $Q$ and the chemical reactions, a balance which may lead to a steady state. ${ }^{(9)}$ Of interest here are the steady-state density $\rho_{s s}$ as a function of the rate $Q$, and the underlying spatial patterns of reactants. In the steady state the expressions (2.2) with (2.4), (2.5), (2.7), and (2.8) are still valid, but now one must relate all the quantities to the (steady-state) density rather than time. In the $A+A \rightarrow 0$ 
case the exponent $\gamma$ in (2.8) is, as before, unity. The relations of the other quantities to the density are still given by (2.4) and (2.5), and $R \sim \rho^{\alpha+1}$. Thus, with the balance $Q=R \sim N / \tau$ we obtain the well-known result for $d_{s} \leqslant 2,{ }^{(8,28)}$

$$
Q \sim \rho_{s s}^{1+2 / d_{s}}
$$

In our earlier work ${ }^{(9)}$ we observed the fact that this result is "the same" as embodied in Eq. (3.2) with the replacement $t^{-\alpha} \rightarrow \rho_{s s}$, and interpreted this to perhaps imply that the spatial distributions of reactants in the two cases is essentially the same. ${ }^{(28)}$ This equality is not self-evident, since the random sources themselves do not reflect this spatial distribution.

In the $A+B \rightarrow 0$ case it is more difficult to establish an expression for the steady-state density, because now the exponent $\gamma$ in (2.8) is no longer unity and one must therefore specify the density dependence of the linear size $A$ of an aggregate. We argue ${ }^{(9)}$ that for high steady-state densities (which can be achieved if the initial density and/or the injection rate are "sufficiently high" and which probably describes essentially all simulations that have been carried out to date) the injection/reaction balance is essentially a local phenomenon that involves little diffusion. The steady-state balance is then between the local excess of one species over the other (proportional to $\sqrt{Q}$ ) due to the random injection process and the time $\tau$ that it takes each of these particles to reach a nearest neighbor:

$$
\sqrt{Q} \sim c \times \frac{1}{\tau}
$$

where $c$ is a coefficient independent of the steady-state density. This then yields

$$
Q \sim \rho_{s s}^{4 / d_{s}}
$$

in agreement with other findings, both theoretical and numerical. ${ }^{(8,29)}$ These arguments do not extend to the case of a low steady-state density, where diffusion plays an important role in the steady state. In this case one might expect a dependence of the steady-state density on the fractal (and/or perhaps other) dimensions in addition to the spectral dimension.

We note that the dependence (4.6) is not "the same" as contained in (2.11), i.e., one cannot be obtained from the other by the simple replacement of $t^{-\alpha}$ by $\rho_{s s}$. We interpret this to indicate that the spatial patterns in the two cases are different. It is possible that this simple replacement may in fact lead to the appropriate result at low densities, i.e., that perhaps for low steady-state densities

$$
Q=\rho_{s s}^{\alpha+1} \sim \rho_{s s}^{1+\left(d_{s} / 2\right)\left[1-\gamma\left(d_{s} / 2 d_{f}\right)\right]}
$$




\section{CONCLUSIONS}

We have revisited our scaling formulation of the problem of diffusionlimited reactions in low-dimensional systems. In particular, we have discussed the anomalous kinetics of $\mathrm{A}+\mathrm{A} \rightarrow 0$ and of $\mathrm{A}+\mathrm{B} \rightarrow 0$ reactions and have recovered all known results from our formulation. We have also presented conjectures as to the behavior of the reaction rates of these processes on fractal geometries in situations where first-principles theories do not yet exist. Our principal result is that the rate of the $A+B \rightarrow 0$ reaction in general depends not only on the spectral dimension, but also on the fractal (and perhaps other) dimensions that characterize the fractal. It is difficult to conclude with finality from numerical results whether our viewpoint is the right one, or whether the theories that lead to a reaction rate that depends only on the spectral dimension are in fact correct. Our results lead to bounds that bracket those of the alternative theories. A definitive conclusion must probably await the calculation of measures that distinguish these approaches more definitively. On the other hand, we find our conceptual reasoning more persuasive at this point.

\section{APPENDIX. RESULTS OF LEYVRAZ AND REDNER FOR EUCLIDEAN GEOMETRIES}

In a recent publication, Leyvraz and Redner ${ }^{(11)}$ introduced a scaling formulation of the $\mathrm{A}+\mathrm{B} \rightarrow 0$ reaction in one- and two-dimensional Euclidean geometries that takes into account the decrease of the reactant density as one approaches the surface of an aggregate relative to the density in its interior, and the related existence of "depletion zones" separating aggregates of unlike particles (a generalization to higher Euclidean dimensions has been suggested by Leyvraz $\left.{ }^{(23)}\right)$. They parametrize the characteristics of the depletion zone (e.g., the density at the boundaries of an aggregate) and fix the parameter so that the known result (3.5) is recovered. It is useful to reproduce their key results so that we can compare our results with theirs and so that me may consider their extension to fractal geometries. We slightly modify their symbols to avoid confusion with notation that we have introduced, and we also drop some subscripts in the interest of simplification.

Leyvraz and Redner consider the properties of single-species aggregates or domains, and introduce the number $n$ of boundary particles per domain. They count a single layer of particles as comprising the boundary. The scaling of $n$ goes as

$$
n \sim\left(\frac{t^{1 / 2}}{l}\right)^{d-1}
$$


where $l$ is the average distance between $\mathrm{A}-\mathrm{A}$ nearest-neighbor pairs on the boundary. The $t^{1 / 2}$ in the numerator is the scaling of the linear size of a segregated domain (which is assumed to have smooth boundaries), a scaling appropriate to compact random walks $(d \leqslant 2)$. The distance between $\mathrm{A}-\mathrm{B}$ nearest-neighbor pairs on neighboring aggregates is also assumed to be $l$. The concentration $\rho_{\mathrm{AB}}$ of boundary particles is the number of boundary particles per unit volume:

$$
\begin{aligned}
\rho_{\mathrm{AB}} & \sim n \times \frac{\text { number of domains }}{\text { volume }}=\frac{n}{\text { volume of a domain }} \sim \frac{n}{t^{d / 2}} \\
& \sim t^{-1 / 2} l^{-(d-1)}
\end{aligned}
$$

The distance $l$ grows with time in an undetermined way, expressed as

$$
l \sim t^{\zeta}
$$

where $\zeta$ is an exponent that they seek to determine. The corresponding distance between like particles in the interior of an aggregate grows as $t^{1 / 4}$, a consequence of the assumption that the distance is proportional to $\rho^{-1 / d}$ and that the density in turn is related to time via Eq. (3.5).

The reaction rate according to Leyvraz and Redner is given by

$$
R \sim \frac{\rho_{\mathrm{AB}}}{l^{2}}
$$

whose denominator is the time required to cover the distance $l$. Combining these results gives for the reaction rate

$$
R \sim t^{-1 / 2} l^{-(d+1)}
$$

With the scaling (A3) one has

$$
R \sim t^{-1 / 2-\zeta(d+1)}
$$

The value of $\zeta$ can be fixed with the knowledge that for Euclidean geometries with $d \leqslant 4$ the rate of the reaction is $R \sim t^{-1-d / 4}$ :

$$
1+\frac{d}{4}=\frac{1}{2}+\zeta(d+1)
$$

In one dimension one obtains $\zeta=3 / 8$ and in two dimensions $\zeta=1 / 3$. In three dimensions these arguments must be modified because the random walk is not compact. Leyvraz ${ }^{(23)}$ concludes that in three dimensions $\zeta=1 / 4$, i.e., there is no depletion zone. He also shows via numerical simula- 
tions that the boundaries of the aggregates are smooth. Their various numerical simulations indicate that their scaling results appear consistent with those of the simulations.

By construction, it follows that our scaling and that of Leyvraz and Redner give the same results for the exponent $\alpha$ in (2.1). If we (perhaps artificially) evaluate the various quantities of the Leyvraz-Redner theory from our expressions, we find that we have fewer "boundary particles per unit volume" than they do, but that our particles have to cover a shorter distance to react. These two factors precisely compensate in the calculation of the reaction rate.

It is interesting to speculate on extensions of the Leyvraz-Redner approach to fractal geometries. One possible generalization (in the spirit of our own generalization of our procedure, for which they cannot be blamed) might be argued as follows. In place of Eq. (A1) we might now write

$$
n \sim\left(\frac{t^{d_{s} / 2 d_{f}}}{l}\right)^{d_{f}-1}
$$

where the $t$-scaling is the linear distance covered by a random walker in time $t$, i.e., it is the usual generalization of the $t^{1 / 2}$ in a Euclidean geometry. The expression (A2) might be generalized to

$$
\rho_{\mathrm{AB}} \sim \frac{n}{\left(t^{d_{s} / 2 d_{f}}\right)^{d_{f}}}=\frac{n}{t^{d_{s} / 2}} \sim \frac{t^{-d_{s} / 2 d_{f}}}{l^{\left(d_{f}-1\right)}}
$$

The expression for the reaction rate might then be generalized to

$$
R \sim \frac{\rho_{\mathrm{AB}}}{l^{2 d_{f} / d_{s}}} \sim t^{-d_{s} / 2 d_{f}} l^{-d_{f}+1-2 d_{f} / d_{s}}
$$

from which follows the exponent

$$
\alpha=\frac{d_{s}}{2 d_{f}}+\zeta\left(d_{f}-1+\frac{2 d_{f}}{d_{s}}\right)-1
$$

The assumption that the dependence of $l$ on $t$ for $1 \leqslant d_{s} \leqslant 2$ is bounded by the dependence found by Leyvraz and Redner for one- and two-dimensional Euclidean geometries leads to bounds for $\alpha$ that are too large in the sense that they do not include the observed exponents. Thus, for example, for a two-dimensional percolation cluster one finds $0.61 \leqslant \alpha \leqslant 0.75$, whereas simulation results are somewhere around $\alpha=0.36$. If $l$ is interpreted as the chemical distance, ${ }^{(30)}$ which grows more rapidly with $t$, the resulting exponent $\alpha$ is even larger. The observed value $\alpha=0.36$ is obtained with $\zeta=0.27$, indicative of a slight depletion effect. 


\section{ACKNOWLEDGMENTS}

The authors thank P. Argyrakis, A. Blumen, S. Havlin, J. Klafter, and G. Zumofen for helpful discussions. This work was supported in part by U.S. Department of Energy grant DE-FG0386ER13606 (K.L.) and by National Science Foundation grant DMR-8801120 (R.K.).

\section{REFERENCES}

1. R. Kopelman, Science 241:1620 (1988).

2. V. Kuzovkov and E. Kotomin, Rep. Prog. Phys. 51:1479 (1988), and references therein.

3. A. A. Ovchinnikov and Y. G. Zeldovich, Chem. Phys. 28:215 (1978).

4. D. Toussaint and F. Wilczek, J. Chem. Phys. 78:2642 (1983).

5. K. Kang and S. Redner, Phys. Rev. Lett, 52:955 (1984).

6. K. Lindenberg, B. J. West, and R. Kopelman, in Noise and Chaos in Nonlinear Dynamical Systems, S. Capelin and F. Moss, eds. (Cambridge University Press, 1989), and references therein.

7. E. Clément, L. M. Sander, and R. Kopelman, Phys. Rev. A 39:6455, 6466 (1989).

8. E. Clément, L. M. Sander, and R. Kopelman, Phys. Rev. A 39:6472 (1989).

9. W.-S. Sheu, K. Lindenberg, and R. Kopelman, Phys. Rev. A 42:2279 (1990).

10. M. Bramson and J. L. Lebowitz, J. Stat. Phys. 62:297 (1991).

11. F. Leyvraz and S. Redner, Phys. Rev. Lett. 66:2168 (1991).

12. G. Zumofen, J. Klafter, and A. Blumen, Phys. Rev. A 43:7068 (1991).

13. L. W. Anacker and R. Kopelman, Phys. Rev. Lett. 58:289 (1987).

14. Z. Jiang and C. Ebner, Phys. Rev. 41:5333 (1990).

15. P. Argyrakis and R. Kopelman, in Dynamics in Small Confining Systems, J. M. Drake, J. Klafter, and R. Kopelman, eds. (Materials Research Society, Pittsburgh, Pennsylvania 1990), p. 195.

16. P. Argyrakis and R. Kopelman, Phys. Rev. A 41:2114, 2121 (1990); P. Argyrakis and R. Kopelman, Phys. Rev. A, in press.

17. S. Kanno, Prog. Theor. Phys. 79:721, 1330 (1988).

18. J.-C. Lin, C. R. Doering, and D. ben-Avraham, Chem. Phys. 146:355 (1990).

19. C. R. Doering and D. ben-Avraham, Phys. Rev. A 38:3035 (1988).

20. K. Lindenberg, W.-S. Sheu, and R. Kopelman, Phys. Rev. A 43:7070 (1991).

21. B. O'Shaughnessy and I. Procaccia, Phys. Rev. 32:3073 (1985).

22. I. M. Sokolov, Phys. Lett. A 139:403 (1989).

23. F. Leyvraz, preprint.

24. L. Rammal and G. Toulouse, J. Phys. Lett. (Paris) 54:44, L13 (1983).

25. P. Evesque and J. Duran, J. Chem. Phys. 80:3016 (1984); P. W. Klymko and R. Kopelman, J. Phys. Chem. 87:4565 (1983).

26. L. A. Harmon, L. Li, L. W. Anacker, and R. K. Kopelman, Chem. Phys. Lett. 163:463 (1989); L. Li, Ph.D. Thesis, University of Michigan (1989).

27. K. Lindenberg, B. J. West, and R. Kopelman, Phys. Rev. A 42:890 (1990).

28. L. W. Anacker and R. Kopelman, J. Chem. Phys. 81:6402 (1984); L. W. Anacker, R. P. Parson, and R. Kopelman, J. Phys. Chem. 89:4758 (1985).

29. L. W. Anacker and R. Kopelman, Science at the John von Neumann Supercomputer Center 1989 (Consortium for Scientific Computing, Princeton, New Jersey, 1990), p. 29.

30. S. Havlin and D. ben-Avraham, Adv. Phys. 36:695 (1987). 